Article

\title{
Plasma Amino Acids May Improve Prediction Accuracy of Cerebral Vasospasm after Aneurysmal Subarachnoid Haemorrhage
}

\author{
Ernest Jan Bobeff ${ }^{1, *(\mathbb{D})}$, Malgorzata Bukowiecka-Matusiak ${ }^{2}{ }^{\mathbb{D}}$, Konrad Stawiski ${ }^{3}$, Karol Wiśniewski ${ }^{1}$ (D), \\ Izabela Burzynska-Pedziwiatr ${ }^{2}{ }^{(D}$, Magdalena Kordzińska ${ }^{4}$, Konrad Kowalski ${ }^{5}$, Przemyslaw Sendys ${ }^{5}$, \\ Michał Piotrowski ${ }^{1}$, Dorota Szczesna ${ }^{2}$, Ludomir Stefańczyk ${ }^{4}$, Lucyna Alicja Wozniak ${ }^{2}$ (D) \\ and Dariusz Jan Jaskólski ${ }^{1}$ (D)
}

check for updates

Citation: Bobeff, E.J.; BukowieckaMatusiak, M.; Stawiski, K.; Wiśniewski, K.; BurzynskaPedziwiatr, I.; Kordzińska, M.; Kowalski, K.; Sendys, P.; Piotrowski, M.; Szczesna, D.; et al. Plasma Amino Acids May Improve Prediction Accuracy of Cerebral Vasospasm after Aneurysmal Subarachnoid Haemorrhage. J. Clin. Med. 2022, 11, 380. https://doi.org/10.3390/ jcm11020380

Academic Editor: Yukinari Kakizawa

Received: 2 November 2021 Accepted: 5 January 2022

Published: 13 January 2022

Publisher's Note: MDPI stays neutral with regard to jurisdictional claims in published maps and institutional affiliations.

Copyright: () 2022 by the authors. Licensee MDPI, Basel, Switzerland. This article is an open access article distributed under the terms and conditions of the Creative Commons Attribution (CC BY) license (https:// creativecommons.org/licenses/by/ $4.0 /)$.
1 Department of Neurosurgery and Neuro-Oncology, Medical University of Lodz, Barlicki University Hospital, Kopcinskiego St. 22, 90-153 Lodz, Poland; karol.wns@gmail.com (K.W.); mmpiotrowskimd@gmail.com (M.P.); dariusz.jaskolski@umed.lodz.pl (D.J.J.)

2 Department of Structural Biology, Medical University of Lodz, 90-419 Lodz, Poland; malgorzata.bukowiecka-matusiak@umed.lodz.pl (M.B.-M.); izabela.burzynska-pedziwiatr@umed.lodz.pl (I.B.-P.); dorota.szczesna@umed.lodz.pl (D.S.); lucyna.wozniak@umed.lodz.pl (L.A.W.)

3 Department of Biostatistics and Translational Medicine, Medical University of Lodz, Mazowiecka 15 Street, 92-215 Lodz, Poland; konrad.stawiski@gmail.com

4 Department of Radiology, Barlicki Memorial Teaching Hospital, Medical University of Lodz, Kopcinskiego 22 Street, 90-153 Lodz, Poland; magdalena.kordzinska@gmail.com (M.K.); ludomir.stefanczyk@umed.lodz.pl (L.S.)

5 Laboratorium Diagnostyczne Masdiag, ul. Żeromskiego 33, 01-882 Warszawa, Poland; konrad.kowalski@masdiag.pl (K.K.); przemyslaw.sendys@masdiag.pl (P.S.)

* Correspondence: ernestbobeff@gmail.com; Tel.: +48-42-677-6770; Fax: +48-42-677-6781

Abstract: Aneurysmal subarachnoid haemorrhages (aSAH) account for 5\% of strokes and continues to place a great burden on patients and their families. Cerebral vasospasm (CVS) is one of the main causes of death after aSAH, and is usually diagnosed between day 3 and 14 after bleeding. Its pathogenesis remains poorly understood. To verify whether plasma concentration of amino acids have prognostic value in predicting CVS, we analysed data from 35 patients after aSAH (median age 55 years, IQR 39-62; 20 females, 57.1\%), and 37 healthy volunteers (median age 50 years, IQR 38-56; 19 females, 51.4\%). Fasting peripheral blood samples were collected on postoperative day one and seven. High performance liquid chromatography-mass spectrometry (HPLC-MS) analysis was performed. The results showed that plasma from patients after aSAH featured a distinctive amino acids concentration which was presented in both principal component analysis and direct comparison. No significant differences were noted between postoperative day one and seven. A total of 18 patients from the study group (51.4\%) developed CVS. Hydroxyproline (AUC $=0.7042,95 \%$ CI 0.5259-0.8826, $p=0.0248$ ) and phenylalanine (AUC $=0.6944,95 \% \mathrm{CI} 0.5119-0.877, p=0.0368$ ) presented significant CVS prediction potential. Combining the Hunt-Hess Scale and plasma levels of hydroxyproline and phenylalanine provided the model with the best predictive performance and the lowest leave-one-out cross-validation of performance error. Our results suggest that plasma amino acids may improve sensitivity and specificity of Hunt-Hess scale in predicting CVS.

Keywords: aneurysmal subarachnoid haemorrhage; cerebral vasospasm; plasma amino acids; predictors; high performance liquid chromatography-mass spectrometry; Hunt-Hess scale

\section{Introduction}

Aneurysmal subarachnoid haemorrhages (aSAH) account for 5\% of strokes, and characteristically it affects younger people. Unlike other strokes, its case fatality varies 
between low to middle- and high-income countries [1], which possibly reflects differences in medical management substantially contributing to the patients' outcome [2]. Although the crude global incidence has declined in recent decades [3], aSAH continues to place a great burden on both patients and their families.

Cerebral vasospasm (CVS) is one of the main causes of death after aSAH. It is responsible for delayed cerebral ischemia (DCI), an umbrella term which encompasses: symptomatic CVS, delayed ischemic neurologic deficit (DIND), and asymptomatic delayed cerebral infarction. CVS is usually diagnosed between day 3 and 14 after bleeding and is characterised by long-term changes in morphology of arterial walls. The pathogenesis remains poorly understood, despite numerous hypotheses concerning the role of blood components, endothelial dysfunction, and vascular innervation, potentially leading to deleterious contraction of cerebral arterial smooth muscles [4].

Factors influencing prognosis after aSAH have been a long-standing issue that has gained special attention since development of microsurgical techniques [5]. The main role of surgical and endovascular interventions remains prevention from rebleeding, thus, its benefits must be carefully weighed against risks. This notwithstanding, it also facilitates CVS prophylaxis and conservative treatment, including vasodilation and haemodynamic augmentation. In 1968 Hunt and Hess stressed that mortality of patients after aSAH is particularly associated with their condition at the time of surgery [6]. Importantly, they introduced a risk stratification Hunt and Hess $(\mathrm{HH})$ scale for selecting the optimal time for surgical intervention, used to date. According to the recent meta-analysis, patients with $\mathrm{HH}$ grade I have lower risk of developing CVS [7]. In poor-grade aSAH patients urgent surgical clipping provided little benefit considering potential intraoperative injury (i.e., aneurysm rupture, difficult exposure, prolonged temporary clipping, blood loss and transfusions, and intraoperative hyper/hypotension) [8]. The new recommendation endorses endovascular coiling in the elderly, poor-grade aSAH, and aneurysms of the basilar tip [9]. Still, it is recommended that treatment decisions be made together with the patient, after receiving recommendations from the multidisciplinary team [10].

Diagnosis of CVS after aSAH requires presence of both, persisting neurologic deficit with delayed onset and relevant changes in radiological imaging. The accepted ancillary tests include digital subtraction angiography (DSA) and transcranial Doppler ultrasound (TCD), whereas computed tomography angiography (CTA) was claimed to overestimate the degree of stenosis [11]. TCD provides rapid, non-invasive, real-time measures of cerebrovascular function, and therefore it is currently considered a standard of care.

Neuromonitoring is a developing interdisciplinary field that helps in early detection of patients at risk of symptomatic CVS, which is critical to successful treatment. Continuous monitoring of brain function requires invasive procedures aimed at maximization of cerebral blood flow (CBF) [12]. On the other hand, there are some novel and less invasive strategies being studied [13]. By way of illustration, in a recent study we proposed a single non-invasive urine biomarker for early diagnosis and monitoring of DCI [14].

Prevention and treatment of CVS remains a matter of debate. Established approaches are the administration of nimodipine initially intravenously then orally and maintenance of euvolemia, as well as treatment with hemodynamic augmentation therapy or endovascular therapy with vasodilators and angioplasty balloons [8]. A recent study also suggested the potential use of heparin [15].

The main objective of this study was to verify whether concentration of plasma amino acids demonstrate diagnostic and prognostic value in predicting CVS in patients after aSAH. Small molecule metabolites fulfil vital functions throughout the body, and thus constitute a favourable target for biomarker research in various diseases. Some authors suggested that haemorrhagic cerebrovascular disease may induce hypermetabolic state of the organism [16,17]. In particular, surgery for SAH showed to provoke a catabolic response and defective utilization of exogenous nitrogen [18], what raised our suspicion that it might impact plasma levels of amino acids. 


\section{Materials and Methods}

We performed a prospective observational case-control study in patients after aSAH treated at the Department of Neurosurgery, Barlicki University Hospital in 2016 and 2017. The study was carried out in compliance with the Helsinki Declaration and ethics approval was acquired from the Ethics Committee of the Medical University of Lodz.

\subsection{Participants}

The inclusion criteria were as follows: 18 to 70 years of age; presence of aneurysm confirmed on computed tomography angiography (CTA) or digital subtraction angiography (DSA); aSAH grade I-III according to the Hunt-Hess scale; preoperative chest X-ray, resting electrocardiogram and routine laboratory tests within normal limits; written informed consent obtained from the patient. The study group consisted of 35 patients after aSAH, out of 122 cases treated at our institution during the enrolment period. All were managed according to the current standard-of-care guidelines [9] and in each case aneurysm repair was performed within $48 \mathrm{~h}$ of bleeding. In the control group there were 37 age- and sex-matched healthy volunteers. The exclusion criteria for both the study and the control groups were specified as follows: serious systemic disease, malnutrition, altered state of consciousness, presence of malignancy, multiple aneurysms, hydrocephalus with the need for external ventricular drainage, and/or prolonged respiratory support $(>24 \mathrm{~h})$. Treatment outcome was assessed at 3 and 12 months after SAH and reported using the Glasgow Outcome Scale, GOS.

\subsection{Cerebral Vasospasm}

Cerebral vasospasm (CVS) was diagnosed only in case of delayed neurological deterioration (i.e., confusion, decreased level of consciousness, or focal neurologic deficit lasting for at least $1 \mathrm{~h}$ ) after excluding other causes. CVS was assessed by TCD, and the threshold was blood flow velocity exceeding $120 \mathrm{~cm} / \mathrm{s}$ in the middle cerebral artery (MCA), and the Lindegaard ratio (MCA/ICA flow velocity) greater than 3. To confirm CVS we performed DSA. All measurements were obtained by an experienced neuroradiologist (LS).

\subsection{Sample Collection}

Fasting peripheral blood samples were collected into commercially available ethylenediaminetetraacetic acid (EDTA) treated tubes on postoperative day one and seven as part of a routine examination. Cells were removed from plasma by centrifugation for $10 \mathrm{~min}$ at $7000 \times g$. Samples were stored in liquid nitrogen afterwards. The overall duration of this phase was limited to $30 \mathrm{~min}$ to minimize its impact on plasma metabolic profile [19]. Determination of metabolites in the collected material was carried out at the Department of Structural Biology, Medical University of Lodz.

\subsection{Chemicals and Reagents}

The methanol used in the procedure was from Sigma Aldrich (Sigma Aldrich Chemie $\mathrm{GmbH}$, Steinheim, Germany). Solution of internal, deuterated standards for amino acids concentration calculation, were produced by CIL (Cambridge Isotope Laboratories, Andover, Tewksbury, MA, USA). For derivatization by butylation of the carboxyl group of the analyte and formation of the butyl ester, $3 \mathrm{~N} \mathrm{HCl}$ in n-butanol (3N Hydrochloric Acid in 1-Butanol) was used from REGIS TECHNOLOGIES, INC.(Austin Avenue, Morton Grove, IL, USA). Formic acid (FA), acetonitrile (ACN), heptafluorobutyric acid (HFBA) ordered from J. T. Baker (Avantor Performance Materials B.V., Deventer, The Netherlands).

\subsection{Sample Preparation}

$10 \mu \mathrm{L}$ of patient plasma with $10 \mu \mathrm{L}$ internal standard solution were placed in polypropylene deep-well plate. Precipitation and extraction were performed by adding $780 \mu \mathrm{L}$ of $1 \mathrm{~N} \mathrm{HCl}$ in methanol. Internal standard solution contains $125 \mathrm{nmol} / \mathrm{mL}$ Gly labeled and $25 \mathrm{nmol} / \mathrm{mL}$ labeled Ala, Asp, Glu, Leu, Met, Phe, Tyr, Val, Orn, Cit. Samples were mixed 
on an orbital shaker at $600 \mathrm{rpm}$, for $10 \mathrm{~min}$, at RT. Precipitation was additionally improved by incubation at $-20^{\circ} \mathrm{C}$ for $2 \mathrm{~h}$. Samples were centrifuged at $1000 \mathrm{rpm}$ for $5 \mathrm{~min} .50 \mu \mathrm{L}$ of supernatant was transferred to a new 96 well plate with $(250 \mu \mathrm{L}$ total well volume). The contents of the well plate were evaporated to dryness for about 15 min. $25 \mu \mathrm{L} 3 \mathrm{~N} \mathrm{HCl}$ in n-butanol was added to each well. The samples prepared in this way were incubated at $60{ }^{\circ} \mathrm{C}$ for $25 \mathrm{~min}$. Evaporated again to dry for about $15 \mathrm{~min}$. Evaporation residue was dissolved in $200 \mu \mathrm{L} \mathrm{H}_{2} \mathrm{O}$ : $\mathrm{MeOH}(80: 20)+0.1 \%$ FA. The dissolved samples were mixed on a shaker at $600 \mathrm{rpm}$ for $10 \mathrm{~min}$, at RT.

\subsection{HPLC-MS Analysis}

HPLC-MS/MS analyses were performed by ExionLC (AB Sciex) liquid chromatograph equipped with an Exigent autosampler (AB Sciex) coupled with tandem mass spectrometer (4500 QTRAP, AB Sciex) with electrospray ion source (Turbo V, AB Sciex). $2 \mu \mathrm{L}$ of the dissolved sample was injected for analysis into ACE Excel C18 column with dimensions of $2.1 \mathrm{~mm} \times 50 \mathrm{~mm} \times 1.7 \mu \mathrm{m}$. Flow ratio of the column was $0.4 \mathrm{~mL} / \mathrm{min}$ with the temperature $40{ }^{\circ} \mathrm{C} . \mathrm{H}_{2} \mathrm{O}$ with $0.5 \mathrm{mM}$ HFBA and $\mathrm{MeOH}$ in proportion with (1:1) ACN constituted respectively, eluents $\mathrm{A}$ and $\mathrm{B}$. The time and used gradient of the eluents are shown in Table 1.

Table 1. Time and percentages of used eluents during HPLC-MS analysis.

\begin{tabular}{ccc}
\hline Time of Elution & Eluent A & Eluent B \\
\hline $\mathbf{( m i n )}$ & $\mathbf{( \% )}$ & $\mathbf{( \% )}$ \\
\hline 0 & 80 & 20 \\
\hline 7 & 50 & 50 \\
\hline 7.1 & 5 & 95 \\
\hline 9 & 5 & 95 \\
\hline 9.1 & 80 & 20 \\
\hline 11 & 80 & 20 \\
\hline
\end{tabular}

\subsection{Statistics}

Statistical analyses were performed using the Statistica software (Version 13; StatSoft, Inc., Tulsa, OK, USA) and R programming language [20]. Due to the lack of normally distributed data (assessed using the Shapiro-Wilk test), we compared continuous variables between two groups with Mann-Whitney's U test. Wilcoxon signed rank exact test for comparison of paired data. The frequencies of categorical variables were compared using Pearson's chi-square test, chi-square test with Yates' correction or two-tailed Fisher's exact test, when appropriate. For all tests, $p<0.05$ was deemed to be significant. Due to the risk of overfitting associated with an event-to-variable ratio of less than 10 , the multivariate analysis was omitted. The principal component analysis was performed to check for outliers and batch effect.

In predictive model development for CVS, the features were at first preselected based on AUC ROC (area under the receiver operating characteristic) curve. A logistic regression models were developed for significant amino acids, Hunt-Hess score (as reference) and for joint model. The cut-off for the prediction estimate was selected based on maximum value of Youden index. Models were assessed based on their accuracy, sensitivity, specificity, PPV (positive predictive value), NPV (negative predictive value). Lastly, due to lack of external validation group, the overfitting of models was assessed in leave-one-out cross-validation (LOOCV).

\section{Results}

We enrolled 35 patients (20 females, 57.1\%) after aSAH who fulfilled the eligibility criteria. All aneurysms were successfully secured within $48 \mathrm{~h}$ after initial bleed, by surgical clipping ( $n=34,97.1 \%)$ or endovascular coiling $(n=1,2.9 \%$; basilar artery aneurysm). 
The median age was 55 years (IQR 39-62) in the study group and 50 years (IQR 38-56) in the control group. The median Hunt-Hess score at presentation was 3 (IQR 2-3). Good outcome (GOS 4-5) was noted in 18 patients (51.4\%) after 3 months and in 23 patients $(65.7 \%)$ after 12 months. Cerebral vasospasm (CVS) was observed in 18 patients $(51.4 \%)$, and it was usually diagnosed on postoperative day 5 (IQR 4-6). Symptoms of CVS were as follows: disorientation 17 (94.4\%), new or increasing H/A 11 (61.1\%), focal neurological deficit $9(50 \%)$, and lethargy $5(27.8 \%)$. More details about the study group clinical features and measurements are provided in Tables 2 and 3.

Table 2. Study group characteristics.

\begin{tabular}{|c|c|}
\hline Characteristic & $n=35$ \\
\hline Male & $15(42.9 \%)$ \\
\hline Age & $55(39-62)$ \\
\hline Length of stay at the dept. of neurosurgery & $15(10-21)$ \\
\hline \multicolumn{2}{|l|}{ Aneurysm location } \\
\hline Anterior communicating artery & $19(54.3 \%)$ \\
\hline Middle cerebral artery & $9(25.7 \%)$ \\
\hline Internal carotid artery & $2(5.7 \%)$ \\
\hline Posterior communicating artery & $2(5.7 \%)$ \\
\hline Basilar artery & $1(2.9 \%)$ \\
\hline Posterior inferior cerebellar artery & $1(2.9 \%)$ \\
\hline Pericallosal artery & $1(2.9 \%)$ \\
\hline \multicolumn{2}{|l|}{ Clinical state on admission } \\
\hline Hunt-Hess score & $3(2-3)$ \\
\hline 1 & $5(14.3 \%)$ \\
\hline 2 & $11(31.4 \%)$ \\
\hline 3 & $19(54.3 \%)$ \\
\hline Fisher score & $4(2-4)$ \\
\hline WFNS score & $2(1-4)$ \\
\hline GCS score & $14(10-15)$ \\
\hline Nuchal rigidity & $29(82.9 \%)$ \\
\hline Neurological deficit & $3(8.6 \%)$ \\
\hline Surgical clipping & $34(97.1 \%)$ \\
\hline Endovascular coiling & $1(2.9 \%)-B A A$ \\
\hline Cerebral vasospasm CVS & $18(51.4 \%)$ \\
\hline Disorientation & $17(94.4 \%)$ \\
\hline New or increasing headache & $11(61.1 \%)$ \\
\hline Focal neurological signs & $9(50 \%)$ \\
\hline Lethargy & $5(27.8 \%)$ \\
\hline Occurrence of CVS (postoperative day) & $5(4-6)$ \\
\hline \multicolumn{2}{|l|}{ Treatment outcome } \\
\hline GOS at discharge & $4(3-4)$ \\
\hline GOS at discharge $\geq 4$ (good outcome) & $18(51.4 \%)$ \\
\hline GOS at one year & $4(2-4)$ \\
\hline GOS at one year $\geq 4$ (good outcome) & $23(65.7 \%)$ \\
\hline mRS & $2(2-5)$ \\
\hline
\end{tabular}

BAA, basilar artery aneurysm; CVS, vertebral vasospasm; GOS, Glasgow outcome scale; mRS, modified Rankin scale; WFNS, World Federation of Neurosurgical Societies. 
Table 3. Intergroup differences analysis of data of 35 patients after aneurysmal subarachnoid hemorrhage. Blood samples were collected on post-operative day 1 and 7 . All concentrations are given in $\mathrm{nmol} / \mathrm{mL}$, and were averaged based on two measurements. ${ }^{*}$ For all tests, $p<0.05$ was deemed to be significant.

\begin{tabular}{|c|c|c|c|c|c|c|c|}
\hline & $\begin{array}{l}\text { Poor Outcome } \\
\text { after } 12 \text { Months } \\
\text { GOS } 1-3, n=12\end{array}$ & $\begin{array}{l}\text { Good Outcome } \\
\text { after } 12 \text { Months } \\
\text { GOS } 4-5, n=23\end{array}$ & $p$ Value & $\begin{array}{c}\text { CVS } \\
n=18\end{array}$ & $\begin{array}{c}\text { No CVS } \\
n=17\end{array}$ & $p$ Value & $\begin{array}{l}\text { Control } \\
\text { Group }\end{array}$ \\
\hline Age & $60.5(36.25-62.75)$ & $52(39-61)$ & 0.424 & $56(46-63.5)$ & $55(33.5-61)$ & 0.215 & $50(38-56)$ \\
\hline Male & $7(58.3 \%)$ & $8(34.8 \%)$ & 0.282 & $10(55.6 \%)$ & $5(29.4 \%)$ & 0.176 & $18(48.6 \%)$ \\
\hline LOS & $18.5(14.5-42.5)$ & $12(9-19)$ & $0.033 *$ & $20.5(15-30.25)$ & $10(8.5-14)$ & $<0.001 *$ & \\
\hline Hunt-Hess score & $3(2.25-3)$ & $2(2-3)$ & 0.140 & $3(2.75-3)$ & $2(1-3)$ & $0.002 *$ & \\
\hline Fisher score & $4(4-4)$ & $3(2-4)$ & $0.009 *$ & $4(3-4)$ & $3(1.5-4)$ & $0.004 *$ & \\
\hline WFNS score & $4(2-4)$ & $1(1-4)$ & 0.051 & $4(2-4)$ & $1(1-2)$ & $0.003 *$ & \\
\hline GCS score & $11(9-13.8)$ & $15(12-15)$ & $0.026^{*}$ & $11.5(9-14)$ & $15(14-15)$ & $0.004^{*}$ & \\
\hline Nuchal rigidity & $11(91.7 \%)$ & $18(78.3 \%)$ & 0.640 & $17(94.4 \%)$ & $12(70.6 \%)$ & 0.088 & \\
\hline $\begin{array}{c}\text { Neurological } \\
\text { deficit }\end{array}$ & $3(25 \%)$ & 0 & $0.034^{*}$ & $2(11.1 \%)$ & $1(5.9 \%)$ & 1.000 & \\
\hline POD-1 Alanine & $153.3(103.7-186.4)$ & $156.8(120-233.4)$ & 0.362 & $151(105.3-192.3)$ & $162.2(126.1-226.3)$ & 0.287 & $\begin{array}{c}266.6 \\
(206.3-311.8)\end{array}$ \\
\hline POD-7 Alanine & $186.3(109.4-249.5)$ & $140.6(109.7-163.5)$ & 0.132 & 120.9 (103.9-218.4) & $147.4(113.7-175.5)$ & 0.636 & \\
\hline POD-1 Arginine & $39.9(35.1-45.3)$ & $37.1(32-50.5)$ & 0.824 & $41.4(34.6-50.1)$ & $36.8(30.5-47.6)$ & 0.245 & $\begin{array}{c}39.6 \\
(32.8-57.3)\end{array}$ \\
\hline POD-7 Arginine & $48.1(36.3-53.8)$ & $39.1(30.8-48.6)$ & 0.151 & $47.8(39-51.9)$ & $36.9(28.2-46.4)$ & $0.038 *$ & \\
\hline POD-1 Asparagine & $17.6(15.7-22.6)$ & $19.2(16.7-22)$ & 0.461 & $18.8(16.5-24.3)$ & $18.5(15.8-21.3)$ & 0.590 & $\begin{array}{c}19.2 \\
(13.6-26.4)\end{array}$ \\
\hline POD-7 Asparagine & $22.7(17.1-25.9)$ & $19.8(16.6-21.6)$ & 0.420 & $20.8(16.8-25.6)$ & $19.6(16.6-23.8)$ & 0.708 & \\
\hline POD-1 Glutamine & $938.3(823.9-1168.5)$ & $960.9(867.1-1340.5)$ & 0.668 & 1053.7 (829.7-1345.3) & 924.5 (875.2-1107.9) & 0.318 & $\begin{array}{c}915.8(774.1- \\
1071.1)\end{array}$ \\
\hline POD-7 Glutamine & 1163.9 (859.5-1301.4) & 1094 (963.2-1238.6) & 0.771 & 1048.8 (935.7-1301.4) & 1161.1 (952.9-1247.1) & 0.757 & \\
\hline $\begin{array}{l}\text { POD-1 Glutamic } \\
\text { acid }\end{array}$ & $41.7(38.8-56.6)$ & $50.7(39.2-62.7)$ & 0.503 & $44(38.3-60.9)$ & $42.1(39.7-61.1)$ & 0.782 & $\begin{array}{c}71.2 \\
(61.2-92.5) \\
\end{array}$ \\
\hline $\begin{array}{l}\text { POD-7 Glutamic } \\
\text { acid }\end{array}$ & $51.2(38.7-61.9)$ & $47.8(37.9-58.6)$ & 0.572 & $53(41.9-65.9)$ & $46.5(34.9-55.8)$ & 0.103 & \\
\hline POD-1 Glycine & $102.1(86.4-134.6)$ & $107.6(91.6-131.4)$ & 0.932 & $114.9(98.4-134.4)$ & $93.9(83-125.5)$ & 0.126 & $\begin{array}{c}178.8 \\
(156.8-215.6)\end{array}$ \\
\hline POD-7 Glycine & $140.2(90.7-196.3)$ & 111.9 (89.1-146.4) & 0.362 & $140.2(99.9-171.8)$ & $100.1(83.4-144.8)$ & 0.057 & \\
\hline POD-1 Isoleucine & $28.6(21-35.5)$ & $34.6(22.8-44.5)$ & 0.461 & $33.9(23.7-44.7)$ & $31(21.5-39.5)$ & 0.424 & $36(23.7-44.7)$ \\
\hline POD-7 Isoleucine & $30.4(23.9-47.6)$ & $38.2(28.7-48.2)$ & 0.440 & $37.2(26.3-52.9)$ & $35.2(27.6-44.5)$ & 0.568 & \\
\hline POD-1 Leucine & $96.4(79.2-157.8)$ & $105.6(78.3-124.2)$ & 0.905 & $101.5(83.5-130.1)$ & $105.6(71.3-132)$ & 0.935 & $\begin{array}{c}138.1 \\
(102.2-175.3)\end{array}$ \\
\hline POD-7 Leucine & $97.1(88.8-126.9)$ & $94.6(80.8-126.7)$ & 0.824 & $98.1(88.6-135.7)$ & $94.6(76.5-120.3)$ & 0.369 & \\
\hline POD-1 Lysine & $69.7(59-83.3)$ & $69.3(61.6-78.2)$ & 0.905 & $74.6(64.5-82.5)$ & $64.4(55.4-74.1)$ & 0.110 & $75.4(56.7-87)$ \\
\hline POD-7 Lysine & $80.5(53.6-90)$ & $72.8(57.2-86.7)$ & 0.771 & $76.3(61-87.8)$ & $78.6(57.2-92.3)$ & 0.807 & \\
\hline POD-1 Methionine & $12.5(10.1-18.8)$ & $11.9(9.1-18.2)$ & 0.851 & $12.5(9.5-19.2)$ & $11.9(9.7-18.1)$ & 0.961 & $17(13.7-20.2)$ \\
\hline POD-7 Methionine & $12.8(11-17.6)$ & $11.2(9.1-13.9)$ & 0.073 & $12.8(10.6-17.3)$ & $11.2(8.9-13)$ & $0.032 *$ & \\
\hline $\begin{array}{c}\text { POD-1 } \\
\text { Phenylalanine }\end{array}$ & $37.6(24.3-45.3)$ & $39.2(31-42.6)$ & 0.824 & $42(32.3-53.6)$ & $33.5(28.5-40.1)$ & $0.049 *$ & $\begin{array}{c}51.2 \\
(8.4-65.3) \\
\end{array}$ \\
\hline $\begin{array}{c}\text { POD-7 } \\
\text { Phenylalanine }\end{array}$ & $44.2(22.7-63.4)$ & $40.9(34-49.7)$ & 0.503 & $46.1(32.7-55.9)$ & $39.7(33.6-44.9)$ & 0.184 & \\
\hline POD-1 Proline & $47.2(39.6-76.3)$ & $62.2(53.6-91)$ & 0.068 & $64.5(45.4-91.5)$ & $58.5(48.2-66.4)$ & 0.660 & $\begin{array}{c}85.6 \\
(63.6-101.6)\end{array}$ \\
\hline POD-7 Proline & $67.7(50.3-125.1)$ & $71.6(54.5-85.5)$ & 0.878 & $69.4(51.2-81.3)$ & $71.6(53-89.4)$ & 0.708 & \\
\hline $\begin{array}{c}\text { POD-1 } \\
\text { Hydroxyproline }\end{array}$ & $2.7(2-3.9)$ & $2.9(2-4.3)$ & 0.440 & $2.1(1.9-4.2)$ & $3.2(2.4-5.1)$ & $0.038^{*}$ & $4.5(2.4-7.3)$ \\
\hline $\begin{array}{c}\text { POD-7 } \\
\text { Hydroxyproline }\end{array}$ & $2.6(0.8-3.4)$ & $3.2(0.2-4.3)$ & 0.440 & $2.2(0.2-3.3)$ & $3.6(2.3-4.5)$ & 0.053 & \\
\hline
\end{tabular}


Table 3. Cont.

\begin{tabular}{|c|c|c|c|c|c|c|c|}
\hline & $\begin{array}{l}\text { Poor Outcome } \\
\text { after } 12 \text { Months } \\
\text { GOS } 1-3, n=12\end{array}$ & $\begin{array}{l}\text { Good Outcome } \\
\text { after } 12 \text { Months } \\
\text { GOS } 4-5, n=23\end{array}$ & $p$ Value & $\begin{array}{c}\text { CVS } \\
n=18\end{array}$ & $\begin{array}{c}\text { No CVS } \\
n=17\end{array}$ & $p$ Value & $\begin{array}{l}\text { Control } \\
\text { Group }\end{array}$ \\
\hline POD-1 Serine & $39.3(31.5-53.9)$ & $42.2(34.5-51.4)$ & 0.878 & $42.3(36.8-53.4)$ & $35.8(30.4-51)$ & 0.184 & $\begin{array}{c}46.2 \\
(31.9-57.5)\end{array}$ \\
\hline POD-7 Serine & $47.8(34.1-58.8)$ & $43.9(39.5-52.2)$ & 0.986 & $48.3(40.4-59.3)$ & $43.7(36.6-52.1)$ & 0.335 & \\
\hline POD-1 Threonine & $32(22.8-39.6)$ & $34.7(25.7-40.7)$ & 0.461 & $35.1(29.4-41.2)$ & $27.8(24.9-39.5)$ & 0.195 & $\begin{array}{c}35.7 \\
(25.8-48.6)\end{array}$ \\
\hline POD-7 Threonine & $33.8(26.5-45.7)$ & $33.6(28.2-43)$ & 0.878 & $36.7(28.9-46.2)$ & $31.9(26.7-42.7)$ & 0.386 & \\
\hline POD-1 Tryptophan & $23.6(21.6-26.1)$ & $26.5(23.7-31.2)$ & 0.172 & $23.7(21.2-31.4)$ & $26.5(25.7-29.6)$ & 0.303 & $\begin{array}{c}30.7 \\
(19.9-37.8)\end{array}$ \\
\hline POD-7 Tryptophan & $22.9(18.3-31.8)$ & $23.9(18.2-33.1)$ & 0.986 & $24(18.5-31.1)$ & $22.8(18.2-36.1)$ & 0.757 & \\
\hline POD-1 Tyrosine & $31.3(25.3-40.6)$ & $29.5(24.8-36.6)$ & 0.771 & $32.9(26.1-41.5)$ & $27.3(24.6-32.5)$ & 0.118 & $42(35.8-52.4)$ \\
\hline POD-7 Tyrosine & $33.9(27.4-37.8)$ & $29.5(24.2-37.8)$ & 0.420 & $33.6(29.3-38.3)$ & $27.7(20.7-37.8)$ & 0.118 & \\
\hline POD-1 Valine & 108.4 (99.9-180.3) & 126.7 (93.7-151.3) & 0.797 & $114(94.2-149.9)$ & $128(93.8-153.7)$ & 0.287 & $\begin{array}{c}167.6 \\
(132.8-203.8)\end{array}$ \\
\hline POD-7 Valine & $122.7(103.7-139.9)$ & $121.5(105.4-146.9)$ & 0.932 & $129(107.8-150.6)$ & $119.2(95-138)$ & 0.173 & \\
\hline POD-1 Histidine & $46.7(37.4-56.1)$ & $47.1(45-58.8)$ & 0.400 & $49.2(42.9-58.1)$ & $46.6(42.9-53.5)$ & 0.245 & $51.3(44-67.3)$ \\
\hline POD-7 Histidine & $49.4(39-63.6)$ & $45.2(40.7-60.3)$ & 0.745 & $46.2(40.8-61.6)$ & $45.2(39.2-61.2)$ & 0.782 & \\
\hline RDW-CV [\%CV] & $13.9(13.3-14.75)$ & $12.8(12.5-13.6)$ & $0.016^{*}$ & $13.4(12.6-14.5)$ & $13(12.5-14.3)$ & 0.716 & \\
\hline RDW-CV >14.5 & $4(33.3 \%)$ & $2(8.7 \%)$ & 0.151 & $3(16.7 \%)$ & $3(17.6 \%)$ & 1 & \\
\hline RDW-SD [fL] & $43.4(40.6-48)$ & $41.6(38.5-45)$ & 0.211 & $43.3(40.3-45.6)$ & $41.6(38.5-44.9)$ & 0.373 & \\
\hline $\begin{array}{c}\text { Cerebral } \\
\text { vasospasm }\end{array}$ & $10(83.3 \%)$ & $8(34.8 \%)$ & 0.011 * & $18(100 \%)$ & NA & & \\
\hline CVS (day) & $5(4-6)$ & $5(4.25-6)$ & 1 & $5(4-6)$ & NA & & \\
\hline 12-month GOS & $2(1-2)$ & $4(4-5)$ & $<0.001 *$ & $2.5(1.75-4)$ & $4(4-5)$ & $<0.001$ * & \\
\hline
\end{tabular}

CVS, cerebral vasospasm; GCS, Glasgow coma scale; GOS, Glasgow outcome scale; LOS, length of stay in hospital; POD, postoperative day; WFNS, World Federation of Neurosurgical Societies.

All evaluated scales (i.e., Hunt-Hess scale, Fisher scale, World Federation of Neurosurgical Societies scale (WFNS) and Glasgow coma scale (GCS)), differed between patients with and without CVS (all $p<0.01$ ). However, only Fisher Scale and GCS differed between patients with poor and good outcome (both $p<0.05$ ). Patients with poor outcome had significantly higher RDW-CV on admission $(13.9 \%$ vs. $12.8 \%, p=0.016)$. Characteristically, patients with CVS were hospitalized for twice as long $(p<0.001)$.

Differential analysis has shown that patients after aSAH, in comparison to healthy controls, featured distinctive amino acids concentration, which was not only shown in the principal component analysis (Figure 1A), but also after the direct comparisons (Figure 1B, Table 4). Interestingly, the concentration of 8 out of 19 studied amino acids was found to be significantly lower in SAH patients, and this remained true after the multiple comparisons' adjustment. No significant differences were noted between postoperative days one and day seven in the SAH patients (Figure 1C,D), indicating a lack of plasma amino acids concentration change during the first seven days after SAH.

Standardized concentration values measured were shown as heatmap in Figure 2. Correlation matrix of concentration was visualized in Figure 3.

In the patients who developed CVS, hydroxyproline (Pro-OH) concentration was significantly lower on postoperative day one (Figure 4B). Arginine and methionine concentration was higher in those patients on postoperative day seven, but all those differences became insignificant after the multiple comparison adjustment. Generally, no significant differences in the amino acids concentration were noted between patients with and without CVS (Figure 4A,B) as well as good and poor GOS result (Figure 4C,D). However, hydroxyproline (Pro-OH, AUC $=0.7042,95 \% \mathrm{CI} 0.5259-0.8826, p=0.0248$ ) and phenylalanine (Phe, AUC $=0.6944,95 \%$ CI $0.5119-0.877, p=0.0368$ ) presented significant CVS prediction potential, as shown in Figure 5. 
Table 4. Differential concentration of amino acids in comparison without and with Benjamini-Hochberg correction of $p$-values. Concentrations were compared using U Mann-Whitney test.

\begin{tabular}{|c|c|c|c|c|c|c|c|c|c|c|c|c|c|c|c|c|c|c|}
\hline \multirow[b]{2}{*}{ Amino Acid } & \multicolumn{3}{|c|}{ POD 1 vs. Controls } & \multicolumn{3}{|c|}{ POD 1 vs. POD 7} & \multicolumn{3}{|c|}{ POD 1 CVS vs. No CVS } & \multicolumn{3}{|c|}{ POD 1 GOS 4-5 vs. GOS 1-3 } & \multicolumn{3}{|c|}{ POD 7 CVS vs. No CVS } & \multicolumn{3}{|c|}{ POD 7 GOS 4-5 vs. GOS 1-3 } \\
\hline & FC & $p$-Value & $\begin{array}{c}\text { Adjusted } \\
p \text {-Value }\end{array}$ & $\begin{array}{c}\text { Median } \\
\text { Difference } \\
{[\text { [nmol/mL] }}\end{array}$ & $p$-Value & $\begin{array}{c}\text { Adjusted } \\
p \text {-Value }\end{array}$ & FC & $p$-Value & $\begin{array}{c}\text { Adjusted } \\
p \text {-Value }\end{array}$ & FC & $p$-Value & $\begin{array}{c}\text { Adjusted } \\
p \text {-Value }\end{array}$ & FC & $p$-Value & $\begin{array}{c}\text { Adjusted } \\
p \text {-Value }\end{array}$ & FC & $p$-Value & $\begin{array}{l}\text { Adjusted } \\
p \text {-Value }\end{array}$ \\
\hline Alanine & 0.7016 & $<0.0001$ & 0.0001 & -15.085 & 0.158359 & 0.77752 & 1.0049 & 0.2834 & 0.5563 & 1.2351 & 0.357 & 0.9446 & 1.086 & 0.6321 & 0.8044 & 0.732 & 0.1305 & 0.9445 \\
\hline Arginine & 0.9326 & 0.7868 & 0.8489 & 2.06 & 0.827161 & 0.899814 & 1.2021 & 0.2413 & 0.5563 & 0.9723 & 0.8213 & 0.9446 & 1.259 & 0.0407 & 0.2739 & 0.8293 & 0.1491 & 0.9445 \\
\hline Asparagine & 0.9613 & 0.7058 & 0.8382 & 1.955 & 0.403521 & 0.77752 & 1.0711 & 0.5974 & 0.8731 & 0.9545 & 0.4654 & 0.9446 & 1.0433 & 0.7042 & 0.8044 & 0.9333 & 0.414 & 0.9861 \\
\hline Glutamine & 1.1165 & 0.0932 & 0.1667 & 142.95 & 0.394466 & 0.77752 & 1.0538 & 0.3221 & 0.5563 & 1.0508 & 0.664 & 0.9446 & 1.0099 & 0.7538 & 0.8044 & 0.9717 & 0.7809 & 0.9861 \\
\hline Glutamic acid & 0.6656 & $<0.0001$ & 0.0001 & 5.41 & 0.576427 & 0.84247 & 1.2119 & 0.7917 & 0.9553 & 1.1815 & 0.4979 & 0.9446 & 1.2825 & 0.1057 & 0.37 & 0.8592 & 0.5663 & 0.9861 \\
\hline Glycine & 0.6447 & $<0.0001$ & $<0.0001$ & 23.055 & 0.294711 & 0.77752 & 1.2596 & 0.1248 & 0.4741 & 0.871 & 0.9446 & 0.9446 & 1.2997 & 0.0577 & 0.2739 & 0.8403 & 0.3569 & 0.9861 \\
\hline Isoleucine & 0.9199 & 0.558 & 0.7067 & 3.825 & 0.334075 & 0.77752 & 1.1715 & 0.4283 & 0.6781 & 1.1799 & 0.4549 & 0.9446 & 1.1392 & 0.5634 & 0.8044 & 1.0318 & 0.4444 & 0.9861 \\
\hline Leucine & 0.8353 & 0.0154 & 0.0372 & -9.54 & 0.852456 & 0.899814 & 1.1543 & 0.9474 & 1 & 0.9827 & 0.917 & 0.9446 & 1.1902 & 0.3639 & 0.6748 & 0.9325 & 0.8348 & 0.9861 \\
\hline Lysine & 1.0252 & 0.8969 & 0.8969 & 9.28 & 0.480881 & 0.77752 & 1.1243 & 0.1131 & 0.4741 & 0.9266 & 0.9032 & 0.9446 & 1.0851 & 0.8044 & 0.8044 & 0.9001 & 0.7676 & 0.9861 \\
\hline Methionine & 0.8441 & 0.0157 & 0.0372 & -0.17333 & 0.279851 & 0.77752 & 1.2607 & 0.9605 & 1 & 0.8998 & 0.862 & 0.9446 & 1.4288 & 0.0346 & 0.2739 & 0.705 & 0.0734 & 0.9445 \\
\hline Phenylalanine & 0.8684 & 0.0965 & 0.1667 & 2.195 & 0.265495 & 0.77752 & 1.2684 & 0.0515 & 0.4741 & 1.0539 & 0.8212 & 0.9446 & 1.2032 & 0.1812 & 0.4303 & 0.8549 & 0.4979 & 0.9861 \\
\hline Proline & 0.7968 & 0.0073 & 0.0232 & 12.075 & 0.231797 & 0.77752 & 1.0548 & 0.6559 & 0.8901 & 1.1348 & 0.0681 & 0.9446 & 1.0574 & 0.7165 & 0.8044 & 0.8834 & 0.8894 & 0.9861 \\
\hline Hydroxyproline & 0.614 & 0.0222 & 0.0468 & -0.2 & 0.491065 & 0.77752 & 0.6068 & 0.0407 & 0.4741 & 1.1181 & 0.4342 & 0.9446 & 0.5021 & 0.0513 & 0.2739 & 1.4491 & 0.4237 & 0.9861 \\
\hline Serine & 0.9392 & 0.8042 & 0.8489 & 4.786667 & 0.045642 & 0.77752 & 1.1596 & 0.1813 & 0.5219 & 0.9604 & 0.8757 & 0.9446 & 1.1772 & 0.3301 & 0.6748 & 0.9703 & 0.9861 & 0.9861 \\
\hline Threonine & 0.9521 & 0.4919 & 0.6676 & -0.36667 & 0.451178 & 0.77752 & 1.1531 & 0.1923 & 0.5219 & 0.9899 & 0.4549 & 0.9446 & 1.1285 & 0.3907 & 0.6748 & 0.9516 & 0.8894 & 0.9861 \\
\hline Tryptophan & 0.8354 & 0.1951 & 0.2851 & -3.195 & 0.743223 & 0.882577 & 1.0028 & 0.2983 & 0.5563 & 1.0289 & 0.1697 & 0.9446 & 1.037 & 0.7664 & 0.8044 & 0.9835 & 0.9861 & 0.9861 \\
\hline Tyrosine & 0.7386 & 0.0001 & 0.0003 & 2.625 & 0.668035 & 0.846178 & 1.2137 & 0.1208 & 0.4741 & 1.0028 & 0.781 & 0.9446 & 1.2141 & 0.1168 & 0.37 & 0.9171 & 0.424 & 0.9861 \\
\hline Histidine & 0.9195 & 0.134 & 0.2122 & -1.19 & 0.967753 & 0.967753 & 1.0721 & 0.8045 & 0.9553 & 1.0368 & 0.3945 & 0.9446 & 1.0926 & 0.7917 & 0.8044 & 0.8725 & 0.7412 & 0.9861 \\
\hline
\end{tabular}

CVS, cerebral vasospasm; FC, fold change; POD, postoperative day. 

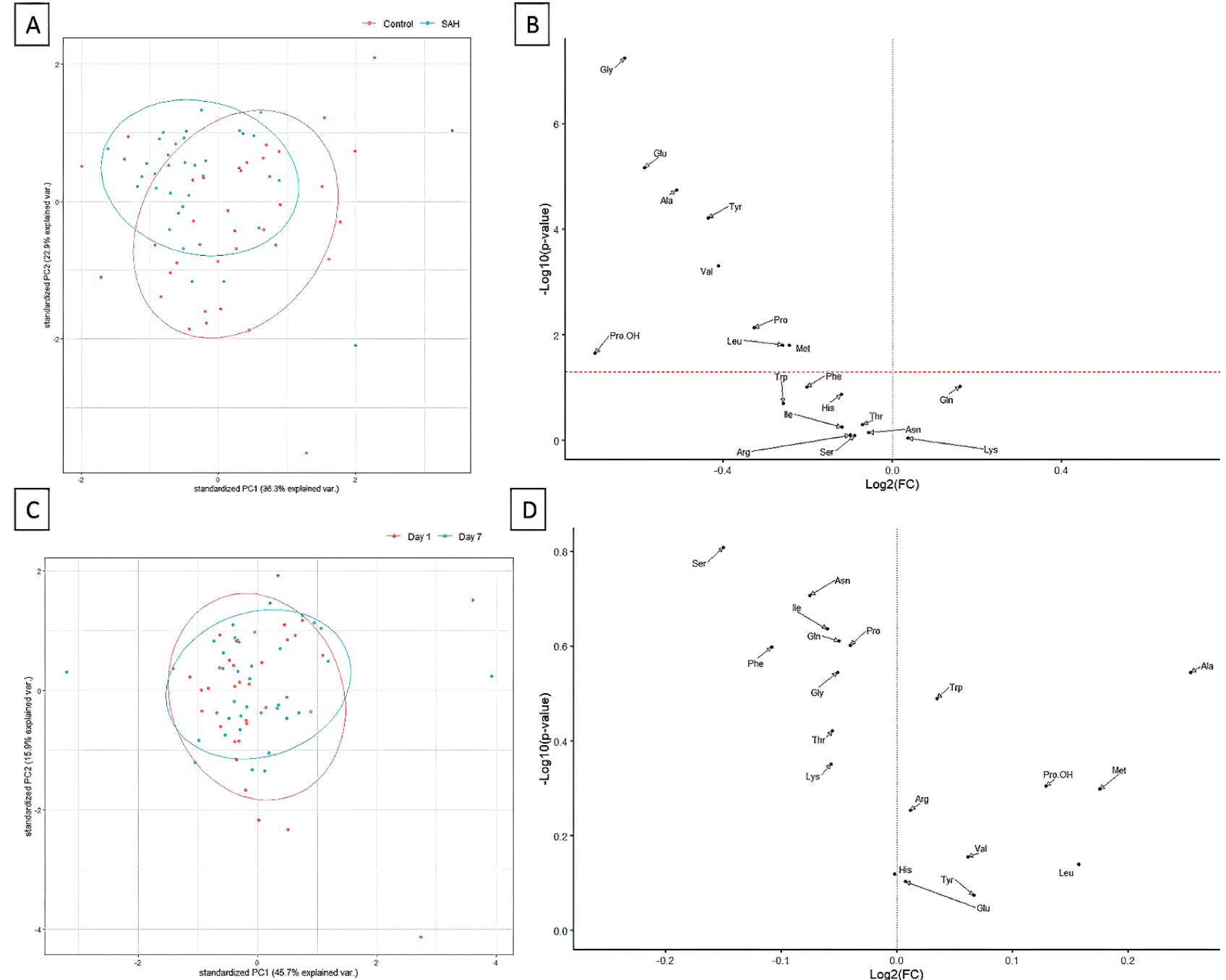

D

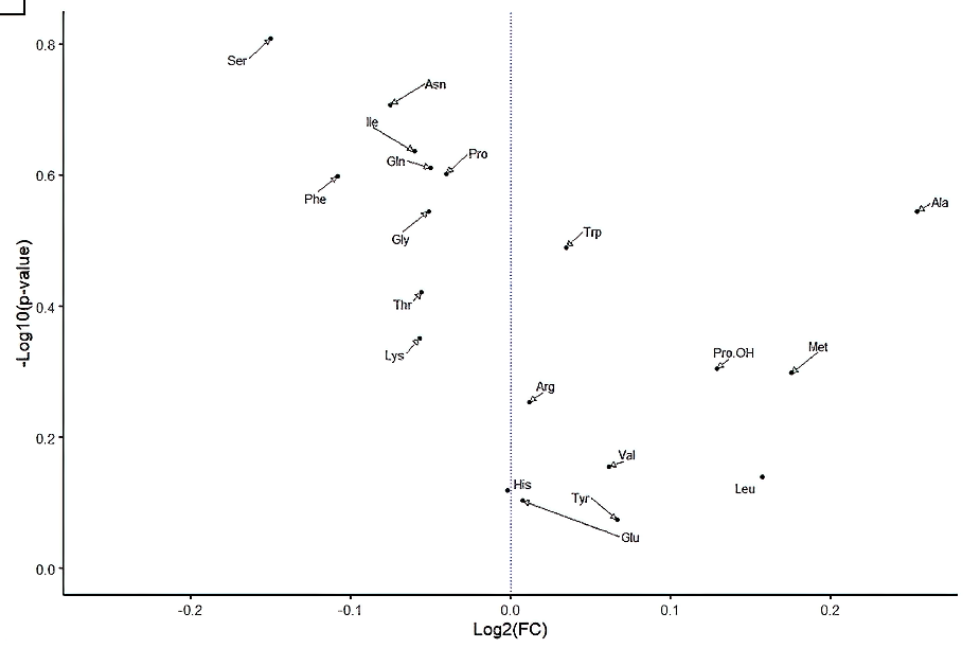

Figure 1. Principal component analysis and differential concentration analysis (volcano plots) showing the differences in amino acids concentration between SAH and control patients $(\mathbf{A}, \mathbf{B})$ and between day one and day seven post-operation (C,D). The relevant $p$-values are available in Table 4 .
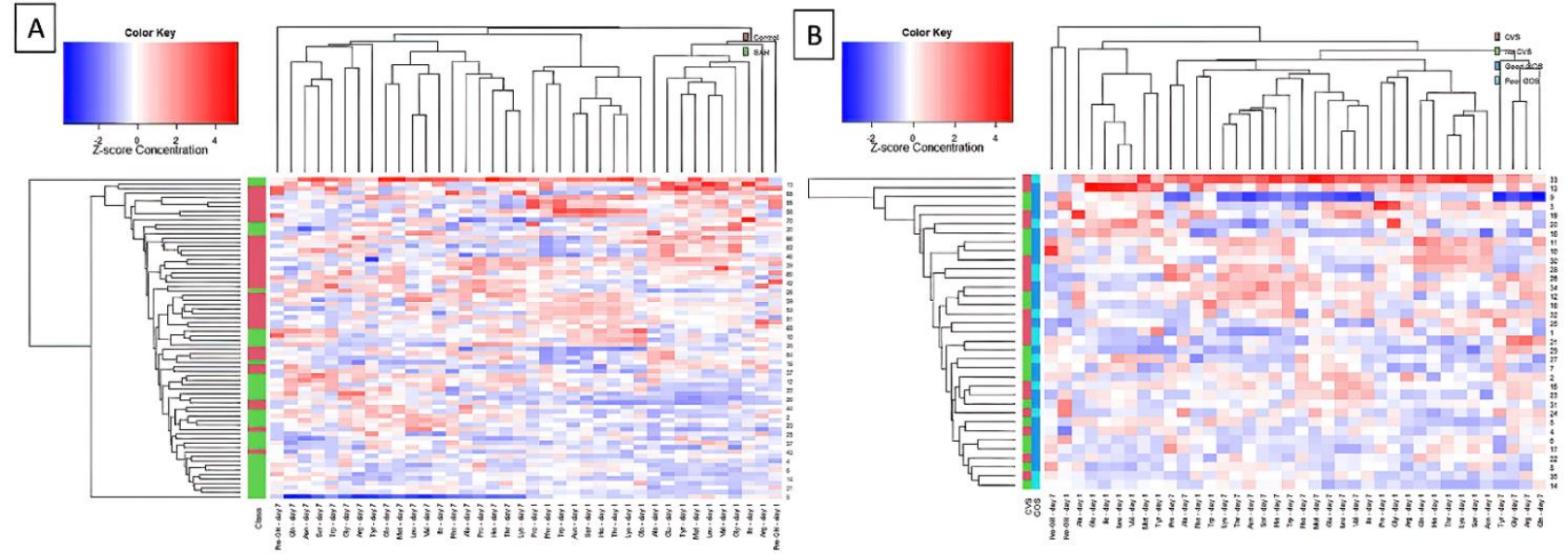

Figure 2. Heatmaps presenting standardized concentration of selected amino acids with hierarchical clustering across both amino acids and samples. (A) presents the comparison between SAH and control patients. SAH patients seem to cluster together. (B) presents intragroup clustering of patients with distinctive GOS and with or without CVS. 
$\leftarrow$

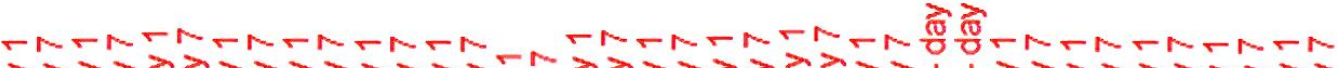

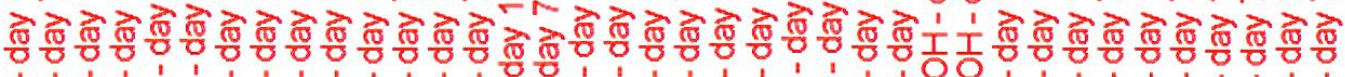

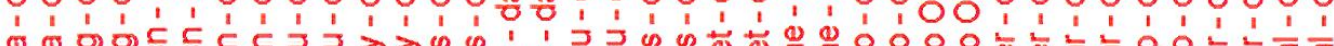

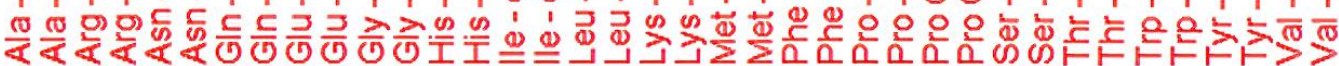

Ala - day 1

Ala - day 7

Arg - day 1

Arg - day 7

Asn - day 1

Asn - day 7

Gln - day 1

Gln - day 7

Glu - day 1

Glu - day 7

Gly - day 1

Gly - day 7

His - day 1

His - day 7

lle - day 1

lle - day 7

Leu - day 1

Leu - day 7

Lys - day 1

Lys - day 7

Met - day 1

Met - day 7

Phe - day 1

Phe - day 7

Pro - day 1

Pro - day 7

Pro $\mathrm{OH}$ - day 1

Pro $\mathrm{OH}$ - day 7

Ser - day 1

Ser - day 7

Thr - day 1

Thr - day 7

Trp - day 1

Trp - day 7

Tyr - day 1

Tyr - day 7

Val - day 1

Val - day 7

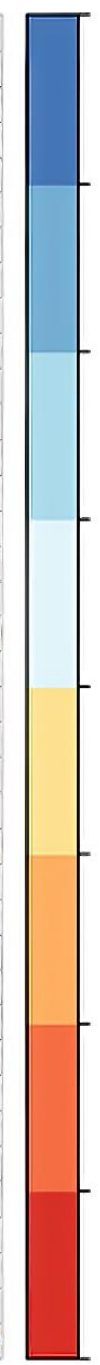

Figure 3. Correlations between amino acids concentration in patients with $\mathrm{SAH}$ and between postoperative day one and day seven. Scale presents the value of $R$ coefficient, indicating the strength of the correlation.

Based on the analysis of area under the ROC curves for selected amino acids and for prediction of CVS (Figure 5) Phe and Pro-OH turned out to be the only amino acids with significant predictive potential. Since the Hunt-Hess scale is considered one of the reference tools for prediction of CVS after SAH, we compared models based on amino acids and Hunt-Hess scale, which performed roughly similar. Combining both the Hunt-Hess scale and amino acids concentration provided the model with the best predictive performance and the lowest leave-one-out cross-validation of performance error, and thus it seemed to be the most resilient model to overfitting (Table 5, Figure 6B). The final logistic regression model presented with excellent accuracy, sensitivity, and specificity, and therefore allowed us to develop a nomogram for prediction of CVS based on the Hunt-Hess scale and plasma concentration of Phe and Pro-OH (Figure 6A). The nomogram can be easily applied in clinical practice and serves for risk stratification of patients beyond established clinical risk markers. 

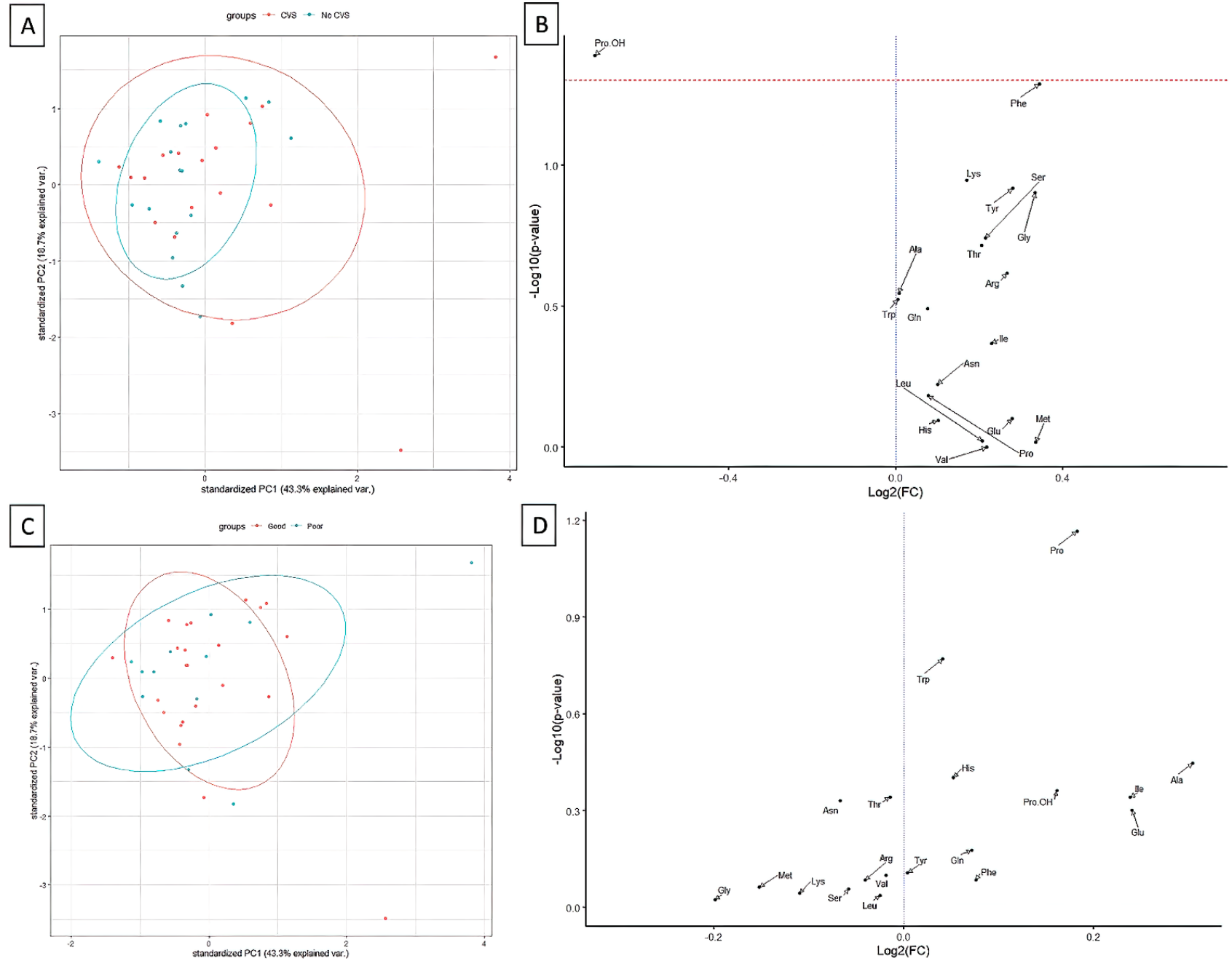

Figure 4. Principal component analysis and differential concentration analysis (volcano plots) showing the differences in amino acids concentration on postoperative day 1 between patients who developed CVS or not $(\mathbf{A}, \mathbf{B})$ and good or bad GOS $(\mathbf{C}, \mathbf{D})$. The relevant $p$-values are available in Table 4.

Phe

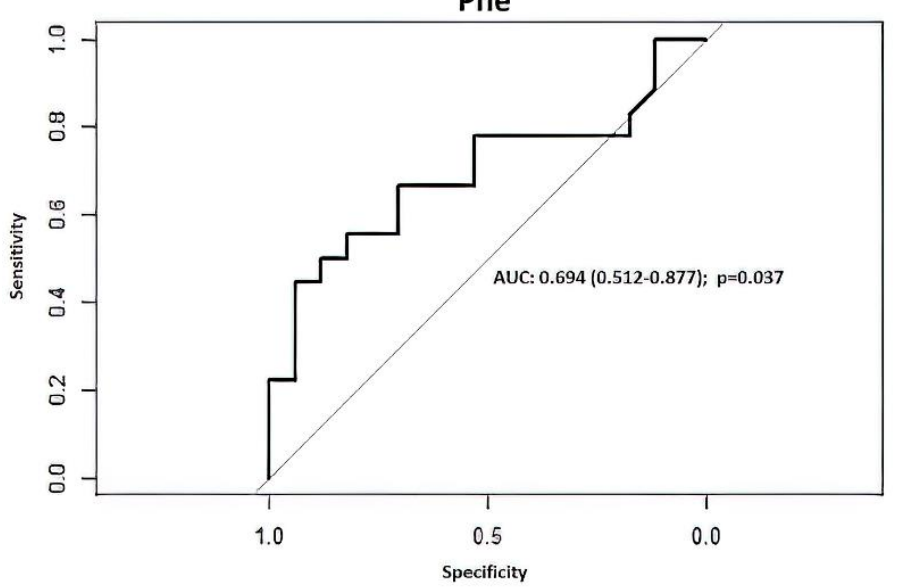

Pro.OH

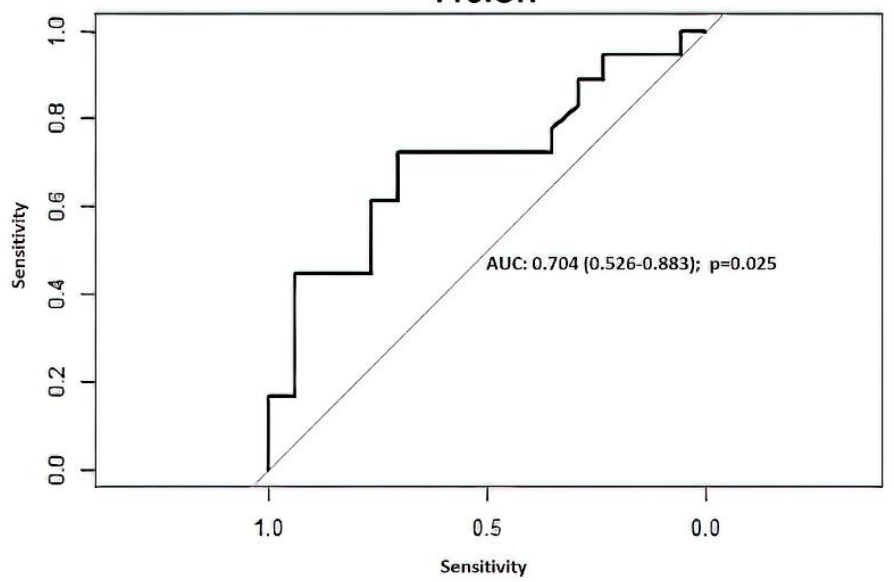

Figure 5. ROC analysis of selected amino acids in prediction of CVS has proved the predictive potential of phenylalanine (Phe) and hydroxyproline (Pro-OH). Area under the curves (AUC) were provided with their $95 \%$ confidence interval in parenthesis. 
Table 5. Performance of logistic regression models.

\begin{tabular}{|c|c|c|c|c|c|c|c|c|c|}
\hline \multirow{4}{*}{$\begin{array}{l}\text { Predictions (Odds } \\
\text { Ratios) }\end{array}$} & \multicolumn{3}{|c|}{ Amino Acids Only (Pro-OH, Phe) } & \multicolumn{3}{|c|}{ Hunt\&Hess Scale Only (HH) } & \multicolumn{3}{|c|}{ Combined Pro-OH, Phe, and HH } \\
\hline & Pro-OH & OR $0.76(9$ & 49-1.18) & \multirow{3}{*}{ HH } & \multirow{3}{*}{\multicolumn{2}{|c|}{ OR 6.84 (95\%CI: 1.78-26.37) }} & Pro-OH & \multicolumn{2}{|c|}{ OR 0.91 (95\%CI: 0.55-1.51) } \\
\hline & \multirow{2}{*}{ Phe } & \multirow{2}{*}{\multicolumn{2}{|c|}{ OR 1.05 (95\%CI: 0.99-1.11) }} & & & & Phe & OR $1.11(9$ & .00-1.23) \\
\hline & & & & & & & HH & OR $15.47(9$ & 30-103.95) \\
\hline \multirow{4}{*}{ Confusion matrix } & \multirow{2}{*}{ Prediction } & \multicolumn{2}{|c|}{ Reference: } & & \multicolumn{2}{|c|}{ Reference: } & & \multicolumn{2}{|c|}{ Reference: } \\
\hline & & No CVS & CVS & Preaiction & No CVS & CVS & Prec & No CVS & CVS \\
\hline & No CVS & 15 & 6 & No CVS & 12 & 4 & No CVS & 14 & 1 \\
\hline & CVS & 2 & 12 & CVS & 5 & 14 & CVS & 3 & 17 \\
\hline Accuracy & \multicolumn{3}{|c|}{ 77.14\% (95\%CI: 59.86-89.56\%) } & \multicolumn{3}{|c|}{ 74.29\% (95\%CI: 56.74-87.51\%) } & \multicolumn{3}{|c|}{$88.57 \%$ (95\%CI: 73.26-96.80\%) } \\
\hline Cutoff & \multicolumn{3}{|c|}{$\geq 0.5455$} & \multicolumn{3}{|c|}{$\geq 0.7529$} & \multicolumn{3}{|c|}{$\geq 0.4283$} \\
\hline Sensitivity & \multicolumn{3}{|c|}{$66.67 \%$} & \multicolumn{3}{|c|}{$77.78 \%$} & \multicolumn{3}{|c|}{$94.44 \%$} \\
\hline Specificity & \multicolumn{3}{|c|}{$88.24 \%$} & \multicolumn{3}{|c|}{$70.59 \%$} & \multicolumn{3}{|c|}{$82.35 \%$} \\
\hline PPV & \multicolumn{3}{|c|}{$85.71 \%$} & \multicolumn{3}{|c|}{$73.68 \%$} & \multicolumn{3}{|c|}{$85.00 \%$} \\
\hline NPV & \multicolumn{3}{|c|}{$71.43 \%$} & \multicolumn{3}{|c|}{$75.00 \%$} & \multicolumn{3}{|c|}{$93.33 \%$} \\
\hline AUC ROC & \multicolumn{3}{|c|}{0.7451 (95\%CI: 0.5619-0.9283) } & \multicolumn{3}{|c|}{0.7745 (95\%CI: 0.6322-0.9168) } & \multicolumn{3}{|c|}{0.9052 (95\%CI: 0.8056-1.0000) } \\
\hline $\begin{array}{l}\text { LOOCV estimate } \\
\text { of prediction error }\end{array}$ & \multicolumn{3}{|c|}{0.2390} & & 0.2015 & & & 0.1797 & \\
\hline
\end{tabular}

AUC, area under the curve; CVS, cerebral vasospasm; $\mathrm{HH}$, Hunt-Hess scale; LOOCV, leave-one-out cross-validation $\mathrm{NPV}$, negative predictive value; positive predictive value; Phe, phenylalanine; Pro-OH, hydroxyproline.

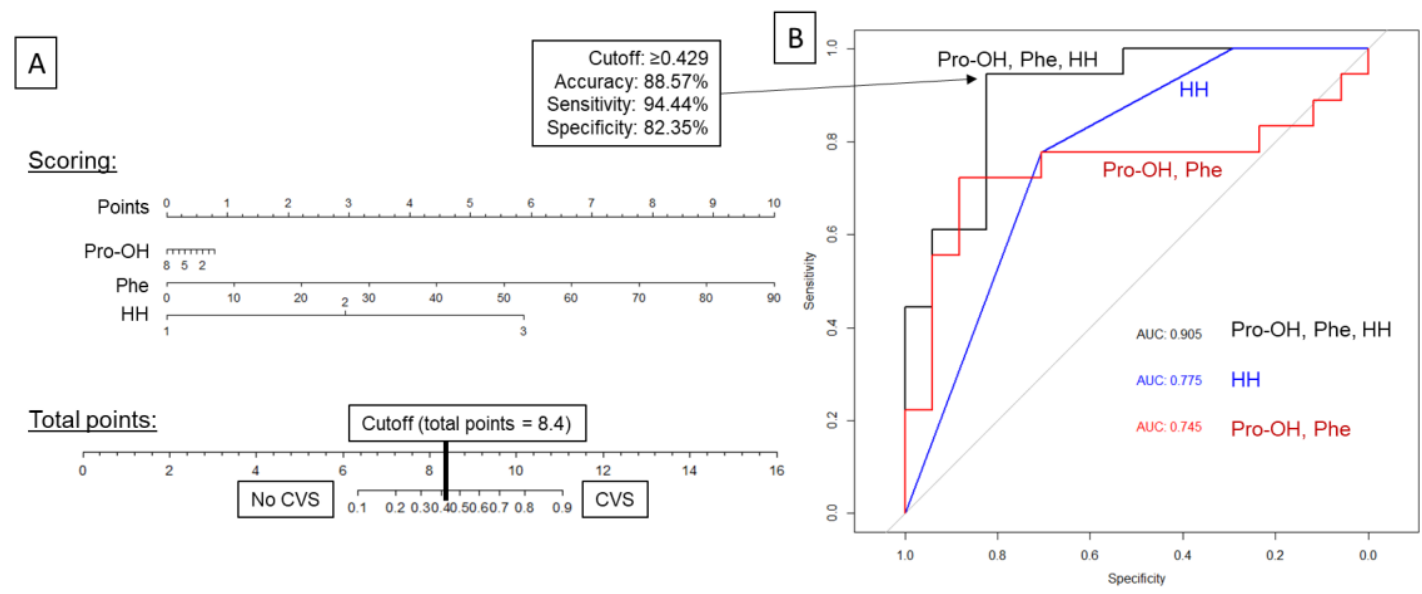

Figure 6. ROC curves of developed logistic regression models with the nomogram for the best model. As shown in (B), the combined model outperformed the model based on amino acids concentration alone (Pro-OH, Phe) and the model based on Hunt-Hess $(\mathrm{HH})$ scale. The best cutoff point for the logistic regression estimate was calculated based on Youden index and marked with arrows and performance characteristics. (A) allows the utilization of the combined model. After scoring individual predictors, if the sum exceeds 8.4, one can predict CVS with a sensitivity of $94.44 \%$ and specificity $82.35 \%$.

\section{Discussion}

Early identification of patients at risk for CVS who could benefit from aggressive intervention is extremely important to improve neurological outcomes. We intended to find a change in the amino acids' concentration in the blood before clinically overt CVS. Hence, the test was conducted just after aneurysm repair and then at the point at which CVS is likely to occur. Our results support the hypothesis that plasma amino acids present diagnostic and predictive value in patients after aSAH. Despite advances in the diagnosis and treatment of aSAH, effective therapeutic interventions are still limited. CVS is one of the major problems responsible for the high mortality and morbidity associated with $\mathrm{SAH}$ [21]. It probably accounts for the impaired cerebral metabolism that has been shown in various studies [22-24]. We reasoned that the metabolites altered in this process could 
be used as a clinical diagnostic tool. Based on the area under the ROC curve analysis, the concentration of hydroxyproline and phenylalanine in the blood on day one after the operation were considered adequate for CVS prognostication, and were further used to increase accuracy of Hunt-Hess scale. The amino acid-based model for CVS prediction in aSAH was comparable to the commonly employed reference tool (i.e., the Hunt-Hess scale). The presented combined model showed the best performance in predicting CVS, and the nomogram provided visualized risk prediction (Table 5, Figures 5 and 6).

What was observed is that both aSAH and surgery induce a hypermetabolic state of the body. One proposed mechanism for this phenomenon is dysregulation of the vegetative system with increased sympathetic activity [16]. Tuoho et al. (1990) found increased resting energy expenditure in patients with haemorrhagic cerebrovascular disease at the acute stage. They also showed that the rate of protein consumption was higher in patients after SAH grade three to four according to Fisher compared to patients in grade one to two. Similarly, Suojaranta-Ylinen et al. (1996) pointed to the effect of adrenergic stimuli on the central nervous system as an underlying factor, and demonstrated that increased amino acid infusion had no effect on amino acid exchange (suggesting that other factors are also involved) [17].

Amino acids are small-molecule metabolites and play an important role in neuronal circuitry, development, maintenance, growth, and survival of neurons in the brain. Molecular movement across the blood-brain barrier is mediated via diffusion, active transport, and carrier-mediated transport, which is particularly used by amino acids. Recently, we demonstrated differences in the levels of certain amino acids in the blood of patients with glioblastoma compared to healthy subjects [25]. The current study, comparing SAH patients with healthy controls, showed that the plasma levels of alanine, glutamic acid, glycine, leucine, methionine, proline, hydroxyproline, tyrosine, and valine differed significantly. Disrupted uptake via blood brain barrier was observed in various brain pathologies including tumours and aSAH [26,27], and it may constitute a common mechanism for the above-mentioned observations. It is interesting to note that there was no significant difference between amino acids concentration on day one and seven post-operation in the SAH group.

Hydroxyproline is a common non-proteinogenic amino acid found predominantly in collagen and elastin-major arterial wall proteins [28]. Unlike other amino acids, it shows smaller biological variation in CSF than in serum [29]. Post-translational modification of hydroxyproline is catalysed by the prolyl subunit of 4-hydroxylase alpha-3 (P4HA3), a key enzyme in collagen synthesis [30]. Increased accumulation of intracellular hydroxyproline and reduced procollagen synthesis in skin fibroblasts were found in patients harbouring cerebral aneurysms [31]. It seems to be consistent with other observations that hydroxyproline and proline are critical for the mechanical strength of collagen [32]. The results of our study demonstrated decreased plasma level of hydroxyproline in patients after aSAH on the postoperative day one compared to controls. In patients who developed CVS later, the level of hydroxyproline was even lower. Similarly, Sokol et al. (2017) reported that the CSF level of hydroxyproline did not increase after aSAH, unlike the rest of amino acids in their group [33]. In an attempt to explain this phenomenon, the authors concluded that the lack of increase in CSF after aSAH might be related to the high binding of hydroxyproline to proteins in human erythrocytes [34]. Presumably, hydroxyproline deficiency can reduce mechanical strength of arterial walls and thus predispose to aneurysm formation and an increased vulnerability to vasospasm. It is possible that in patients with a genetic predisposition, blood levels of hydroxyproline may be a predictor of aSAH as well. Interestingly, the theory that plasma amino acids concentration may be involved in etiopathogenesis of SAH has been already formulated [35,36], although the authors focused on the level of homocysteine, which we did not measure.

Phenylalanine is an essential amino acid involved in protein biosynthesis. It contains a distinctive aromatic ring which makes it one of the biggest proteinogenic amino acid. It is also converted to tyrosine, a precursor of monoamine neurotransmitters. Both phenylala- 
nine and tyrosine are substrates for tyrosine hydroxylase, the enzyme catalysing the ratelimiting step in catecholamine synthesis. The first conversion catalysed by phenylalanine hydroxylase is the source of L-tyrosine, but is also the first step in tyrosine/phenylalanine catabolism. Tyrosine can be converted into 3,4-dihydroxy-L-phenylalanine (L-DOPA), which, in the next step, is converted into dopamine, norepinephrine, and epinephrine [37]. Tyrosine is the preferred substrate; consequently, unless its concentration is abnormally low, changes in phenylalanine concentration does not affect catecholamine synthesis. Unlike tyrosine, phenylalanine does not exhibit substrate inhibition, therefore, its high concentration does not inhibit catecholamine synthesis [37]. Clinical and experimental studies have shown that patients after SAH exhibited significant activation of the sympathetic nervous system [38]. Minegishi et al. (1987) demonstrated that plasma norepinephrine metabolite levels can serve as a prognostic discriminator for patients after aSAH [39]. Catecholamine surge causes activation of the sympathetic nervous system and may induce arrhythmias, neurogenic pulmonary edema, injury to the hypothalamus and brainstem, and is probably associated with the development of CVS [40]. We observed significantly higher levels of phenylalanine on day one post-operation in patients who later developed CVS $(p=0.049)$, what is consistent with the literature cited above.

An important contribution to the understanding of amino acid metabolism in brain pathology was based on studies concerning composition of interstitial fluid (ISF) and cerebrospinal fluid (CSF). Zetterling et al. (2009) reported increased concentration of eight amino acids (alanine, asparagine, glutamine, isoleucine, leucine, phenylalanine, serine, and tyrosine) in ISF after SAH [41]. Li et al. (2019) found that increased amino acid levels in CSF after SAH was associated with unfavorable outcome [42]. Particularly, high levels of asymmetric and symmetric dimethylarginines (ADMA, SDMA) in CSF were associated with poor outcome [43]. The same authors confirmed that plasma L-arginine/ADMA ratio is negatively associated with outcome, which was earlier suggested by Staalsø et al. [44]. Although we did not measure the ratio, our results showed that the absolute value of plasma arginine was higher in patients with poor outcome and those who developed CVS. Von Holst and Hagenfeldt (1985) demonstrated increased amino acids concentration in CSF after SAH and proposed mechanisms leading to this phenomenon [45]. The initial increase may be due to extravasation of blood into the CSF and therefore depends on the amount of bleeding. It also might be due to the increased amino acid turnover in response to injury and proteolysis, which initiates a repair process that is in turn accompanied by increased protein synthesis [46]. The widespread activation often extends beyond the area of actual brain damage [47]. When combined with impaired transport across the blood-brain barrier it results in increased amino acids concentration in CSF and ISF and decreased amino acids concentration in plasma. Interestingly, we also observed decreased levels of amino acids in plasma. Other biological compounds have been studied as well. Bellapart et al. (2014) found that endothelin-1 level is higher in plasma than in CSF on the day five after SAH in patients who developed CVS [48]. On the other hand, CVS occurs on average at that day, whereas the idea of predictive biomarker for CVS is to indicate patients at risk beforehand. In addition, the plasma biomarker would be more desirable since not all of the patients after aSAH require CSF study.

Despite the fact that our results have empirically demonstrated the possible utility of plasma amino acid analysis in patients after aSAH, there are some limitations of this study that can be addressed in developing a new model. First, the proposed measurements are not part of the basic tests performed in a hospital, and thus the results cannot yet be implemented in clinical practice. However, they may provide guidance for subsequent clinical studies. Given the magnitude of disease burden, our sample size remains somewhat inadequate to expect similar results in a population-based study. Predictive models built on small groups may be particularly susceptible to overfitting. One reason for clinicians not using them is the lack of external validation so that they do not know if it predicts the risk precisely in other patients. The main sources of potential bias can be dietary habits, stimulant use, and often under-recognized common chronic diseases. 


\section{Conclusions}

The purpose of the current study was to determine whether plasma amino acids may help to differentiate between patients with and without increased risk of developing CVS after aSAH. The results presented here can potentially be used in a clinical setting along with reference tools. One of the more significant findings to emerge from this study is that plasma levels of hydroxyproline and phenylalanine may improve sensitivity and specificity of Hunt-Hess scale in predicting CVS. It is suggested that the association of these factors should be further investigated.

Author Contributions: Conceptualization, E.J.B.; data curation, E.J.B.; formal analysis, E.J.B., M.B.-M., K.S., and K.W.; funding acquisition, D.J.J.; investigation, E.J.B., M.B.-M., K.S., I.B.-P., and M.K.; methodology, M.B.-M. and I.B.-P.; project administration, E.J.B.; resources, K.K., P.S., and D.S.; supervision, D.J.J.; visualization, M.P.; writing—original draft, E.J.B.; writing-review and editing, L.S., L.A.W., and D.J.J. All authors have read and agreed to the published version of the manuscript.

Funding: This work was supported by Ministry of Science and Higher Education Funding for Young Scientists nr. 502-03/1-121-03/502-14-286.

Institutional Review Board Statement: The study was conducted according to the guidelines of the Declaration of Helsinki, and approved by the local Bioethical Committee at the Medical University of Lodz, number RNN/225/15/KE (6 July 2015).

Informed Consent Statement: Informed consent was obtained from all subjects involved in the study.

Data Availability Statement: The data presented in this study are available on request from the corresponding author. The data are not publicly available due to ethical restrictions.

Conflicts of Interest: The authors declare that the article content was composed in the absence of any commercial or financial relationships that could be construed as a potential conflict of interest.

\section{References}

1. Feigin, V.L.; Lawes, C.M.; Bennett, D.A.; Barker-Collo, S.L.; Parag, V. Worldwide stroke incidence and early case fatality reported in 56 population-based studies: A systematic review. Lancet Neurol. 2009, 8, 355-369. [CrossRef]

2. Ingall, T.; Asplund, K.; Mähönen, M.; Bonita, R. A Multinational Comparison of Subarachnoid Hemorrhage Epidemiology in the WHO MONICA Stroke Study. Stroke 2000, 31, 1054-1061. [CrossRef] [PubMed]

3. Etminan, N.; Chang, H.-S.; Hackenberg, K.; De Rooij, N.K.; Vergouwen, M.D.I.; Rinkel, G.J.E.; Algra, A. Worldwide Incidence of Aneurysmal Subarachnoid Hemorrhage According to Region, Time Period, Blood Pressure, and Smoking Prevalence in the Population. JAMA Neurol. 2019, 76, 588-597. [CrossRef]

4. Jaskólski, D.; Zawirski, M. Pathophysiology of cerebral vasospasm after subarachnoid hemorrhage from ruptured intracranial aneurysm. Pol. Tyg. Lek. 1988, 43, 748-752. [PubMed]

5. Hamby, W.B. Spontaneous Subarachnoid Hemorrhage of Aneurysmal Origin. J. Am. Med Assoc. 1948, 136, 522-528. [CrossRef]

6. Hunt, W.E.; Hess, R.M. Surgical Risk as Related to Time of Intervention in the Repair of Intracranial Aneurysms. J. Neurosurg. 1968, 28, 14-20. [CrossRef] [PubMed]

7. Rumalla, K.; Lin, M.; Ding, L.; Gaddis, M.; Giannotta, S.L.; Attenello, F.J.; Mack, W.J. Risk Factors for Cerebral Vasospasm in Aneurysmal Subarachnoid Hemorrhage: A Population-Based Study of 8346 Patients. World Neurosurg. 2021, 145, e233-e241. [CrossRef]

8. Muirhead, W.R.; Grover, P.J.; Toma, A.K.; Stoyanov, D.; Marcus, H.J.; Murphy, M. Adverse intraoperative events during surgical repair of ruptured cerebral aneurysms: A systematic review. Neurosurg. Rev. 2021, 44, 1273-1285. [CrossRef] [PubMed]

9. Connolly, E.S.; Rabinstein, A.A.; Carhuapoma, J.; Derdeyn, C.; Dion, J.; Higashida, R.T.; Hoh, B.L.; Kirkness, C.J.; Naidech, A.M.; Ogilvy, C.S.; et al. Guidelines for the Management of Aneurysmal Subarachnoid Hemorrhage. Stroke 2012, 43, 1711-1737. [CrossRef]

10. Harris, L.; Hill, C.S.; Elliot, M.; Fitzpatrick, T.; Ghosh, A.; Vindlacheruvu, R. Comparison between outcomes of endovascular and surgical treatments of ruptured anterior communicating artery aneurysms. Br. J. Neurosurg. 2021, 35, 313-318. [CrossRef]

11. Greenberg, E.; Gold, R.; Reichman, M.; John, M.; Ivanidze, J.; Edwards, A.; Johnson, C.; Comunale, J.; Sanelli, P. Diagnostic Accuracy of CT Angiography and CT Perfusion for Cerebral Vasospasm: A Meta-Analysis. Am. J. Neuroradiol. 2010, 31, 1853-1860. [CrossRef] [PubMed]

12. Vajkoczy, P.; Horn, P.; Thome, C.; Munch, E.; Schmiedek, P. Regional cerebral blood flow monitoring in the diagnosis of delayed ischemia following aneurysmal subarachnoid hemorrhage. J. Neurosurg. 2003, 98, 1227-1234. [CrossRef]

13. Przybycien-Szymanska, M.M.; Ashley, W.W. Biomarker Discovery in Cerebral Vasospasm after Aneurysmal Subarachnoid Hemorrhage. J. Stroke Cerebrovasc. Dis. 2015, 24, 1453-1464. [CrossRef] [PubMed] 
14. Wiśniewski, K.; Popęda, M.; Tomasik, B.; Bieńkowski, M.; Bobeff, E.J.; Stefańczyk, L.; Tybor, K.; Hupało, M.; Jaskólski, D.J. The Role of Urine F2-ISOPROSTANE Concentration in Delayed Cerebral Ischemia after Aneurysmal Subarachnoid Haemorrhage-A Poor Prognostic Factor. Diagnostics 2020, 11, 5. [CrossRef] [PubMed]

15. Kole, M.J.; Wessell, A.P.; Ugiliweneza, B.; Cannarsa, G.J.; Fortuny, E.; Stokum, A.J.; Shea, P.; Chryssikos, T.; Khattar, N.K.; Crabill, A.G.; et al. Low-Dose Intravenous Heparin Infusion after Aneurysmal Subarachnoid Hemorrhage is Associated with Decreased Risk of Delayed Neurological Deficit and Cerebral Infarction. Neurosurgery 2021, 88, 523-530. [CrossRef]

16. Touho, H.; Karasawa, J.; Shishido, H.; Morisako, T.; Yamada, K.; Shibamoto, K. Hypermetabolism in the acute stage of hemorrhagic cerebrovascular disease. J. Neurosurg. 1990, 72, 710-714. [CrossRef] [PubMed]

17. Suojaranta-Ylinen, R. Hypermetabolism and Increased Peripheral Release of Amino Acids After Subarachnoidal Hemorrhage and Its Operative Treatment. Nutrition 1996, 12, 327-333. [CrossRef]

18. Hersio, K.; Vapalahti, M.; Kari, A.; Takala, J.; Hernesniemi, J.; Tapaninaho, A.; Luukkonen, M. Impaired utilization of exogenous amino acids after surgery for subarachnoid haemorrhage. Acta Neurochir. 1990, 106, 13-17. [CrossRef]

19. Jobard, E.; Trédan, O.; Postoly, D.; André, F.; Martin, A.-L.; Elena-Herrmann, B.; Boyault, S. A Systematic Evaluation of Blood Serum and Plasma Pre-Analytics for Metabolomics Cohort Studies. Int. J. Mol. Sci. 2016, 17, 2035. [CrossRef]

20. R Core Team. R: A Language and Environment for Statistical Computing; R Foundation for Statistical Computing: Vienna, Austria, 2017. Available online: https:/ / www.R-project.org/ (accessed on 20 July 2021).

21. Dorsch, N.W. Cerebral arterial spasm-a clinical review. Br. J. Neurosurg. 1995, 9, 403-412. [CrossRef]

22. Hayashi, T.; Suzuki, A.; Hatazawa, J.; Hadeishi, H.; Shirane, R.; Tominaga, T.; Yasui, N. Post-operative changes of cerebral circulation and metabolism in the acute stage of low-grade aneurysmal subarachnoid hemorrhage. Neurol. Res. 2008, 30, 678-683. [CrossRef]

23. Hayashi, T.; Suzuki, A.; Hatazawa, J.; Kanno, I.; Shirane, R.; Yoshimoto, T.; Yasui, N. Cerebral circulation and metabolism in the acute stage of subarachnoid hemorrhage. J. Neurosurg. 2000, 93, 1014-1018. [CrossRef]

24. Wagner, M.; Jurcoane, A.; Hildebrand, C.; Güresir, E.; Vatter, H.; Zanella, F.; Berkefeld, J.; Pilatus, U.; Hattingen, E. Metabolic Changes in Patients with Aneurysmal Subarachnoid Hemorrhage Apart from Perfusion Deficits: Neuronal Mitochondrial Injury? Am. J. Neuroradiol. 2013, 34, 1535-1541. [CrossRef]

25. Bobeff, E.J.; Szczesna, D.; Bieńkowski, M.; Janczar, K.; Chmielewska-Kassassir, M.; Wiśniewski, K.; Papierz, W.; Wozniak, L.A.; Jaskólski, D.J. Plasma amino acids indicate glioblastoma with ATRX loss. Amino Acids 2021, 53, 119-132. [CrossRef]

26. Guan, Z.; Lan, H.; Cai, X.; Zhang, Y.; Liang, A.; Li, J. Blood-Brain Barrier, Cell Junctions, and Tumor Microenvironment in Brain Metastases, the Biological Prospects and Dilemma in Therapies. Front. Cell Dev. Biol. 2021, 9. [CrossRef] [PubMed]

27. Suzuki, H.; Kanamaru, H. Potential therapeutic molecular targets for blood-brain barrier disruption after subarachnoid hemorrhage. Neural Regen. Res. 2019, 14, 1138-1143. [CrossRef] [PubMed]

28. Macdonald, R.L.; Weir, B.K.A.; Young, J.D.; Grace, M.G.A. Cytoskeletal and extracellular matrix proteins in cerebral arteries following subarachnoid hemorrhage in monkeys. J. Neurosurg. 1992, 76, 81-90. [CrossRef]

29. Kruse, T.; Reiber, H.; Neuhoff, V. Amino acid transport across the human blood-CSF barrier: An evaluation graph for amino acid concentrations in cerebrospinal fluid. J. Neurol. Sci. 1985, 70, 129-138. [CrossRef]

30. Wang, T.; Wang, Y.-X.; Dong, Y.-Q.; Yu, Y.-L.; Ma, K. Prolyl 4-hydroxylase subunit alpha 3 presents a cancer promotive function in head and neck squamous cell carcinoma via regulating epithelial-mesenchymal transition. Arch. Oral Biol. $2020,113,104711$. [CrossRef]

31. Majamaa, K.; Myllylä, V.V. A disorder of collagen biosynthesis in patients with cerebral artery aneurysm. Biochim. Biophys. Acta BBA-Mol. Basis Dis. 1993, 1225, 48-52. [CrossRef]

32. Kotch, F.W.; Guzei, I.A.; Raines, R.T. Stabilization of the Collagen Triple Helix by O-Methylation of Hydroxyproline Residues. J. Am. Chem. Soc. 2008, 130, 2952-2953. [CrossRef]

33. Sokół, B.; Urbaniak, B.; Wąsik, N.; Plewa, S.; Klupczynska, A.; Jankowski, R.; Więckowska, B.; Juszkat, R.; Kokot, Z. Amino Acids in Cerebrospinal Fluid of Patients with Aneurysmal Subarachnoid Haemorrhage: An Observational Study. Front. Neurol. 2017, 8 , 438. [CrossRef] [PubMed]

34. Siddiqi, N.J.; Alhomida, A.S. Distribution of Total, Free, Peptide-bound and Protein-bound Hydroxyproline in the Erythrocytes from Different Species. Comp. Haematol. Int. 2002, 11, 123-128. [CrossRef]

35. Dhandapani, S.; Goudihalli, S.; Mukherjee, K.K.; Singh, H.; Srinivasan, A.; Danish, M.; Mahalingam, S.; Dhandapani, M.; Gupta, S.K.; Khandelwal, N.; et al. Prospective study of the correlation between admission plasma homocysteine levels and neurological outcome following subarachnoid hemorrhage: A case for the reverse epidemiology paradox? Acta Neurochir. 2014, 157, 399-407. [CrossRef] [PubMed]

36. Kumar, M.; Goudihalli, S.; Mukherjee, K.; Dhandapani, S.; Sandhir, R. Methylenetetrahydrofolate reductase C677T variant and hyperhomocysteinemia in subarachnoid hemorrhage patients from India. Metab. Brain Dis. 2018, 33, 1617-1624. [CrossRef] [PubMed]

37. Fernstrom, J.D.; Fernstrom, M.H. Tyrosine, Phenylalanine, and Catecholamine Synthesis and Function in the Brain. J. Nutr. 2007, 137, 1539S-1547S. [CrossRef] [PubMed]

38. Lambert, G.; Naredi, S.; Edén, E.; Rydenhag, B.; Friberg, P. Monoamine metabolism and sympathetic nervous activation following subarachnoid haemorrhage: Influence of gender and hydrocephalus. Brain Res. Bull. 2002, 58, 77-82. [CrossRef] 
39. Minegishi, A.; Ishizaki, T.; Yoshida, Y.; Ahagon, A.; Shibata, N.; Kobayashi, H. Plasma Monoaminergic Metabolites and Catecholamines in Subarachnoid Hemorrhage. Arch. Neurol. 1987, 44, 423-428. [CrossRef]

40. Hasegawa, Y.; Uchikawa, H.; Kajiwara, S.; Morioka, M. Central sympathetic nerve activation in subarachnoid hemorrhage. J. Neurochem. 2021, 160, 34-50. [CrossRef]

41. Zetterling, M.; Hillered, L.; Samuelsson, C.; Karlsson, T.; Enblad, P.; Ronne-Engström, E. Temporal patterns of interstitial pyruvate and amino acids after subarachnoid haemorrhage are related to the level of consciousness-A clinical microdialysis study. Acta Neurochir. 2009, 151, 771-780. [CrossRef]

42. Li, Y.-C.; Wang, R.; Xu, M.-M.; Jing, X.-R.; Ji-Ye, A.; Sun, R.-B.; Na, S.-J.; Liu, T.; Ding, X.-S.; Sun, C.-Y.; et al. Aneurysmal Subarachnoid Hemorrhage Onset Alters Pyruvate Metabolism in Poor-Grade Patients and Clinical Outcome Depends on More: A Cerebrospinal Fluid Metabolomic Study. ACS Chem. Neurosci. 2019, 10, 1660-1667. [CrossRef] [PubMed]

43. Appel, D.; Seeberger, M.; Schwedhelm, E.; Czorlich, P.; Goetz, A.E.; Böger, R.H.; Hannemann, J. Asymmetric and Symmetric Dimethylarginines are Markers of Delayed Cerebral Ischemia and Neurological Outcome in Patients with Subarachnoid Hemorrhage. Neurocrit. Care 2018, 29, 84-93. [CrossRef]

44. Staalsø, J.M.; Bergström, A.; Edsen, T.; Weikop, P.; Romner, B.; Olsen, N.V. Low Plasma Arginine: Asymmetric Dimethyl Arginine Ratios Predict Mortality after Intracranial Aneurysm Rupture. Stroke 2013, 44, 1273-1281. [CrossRef] [PubMed]

45. Von Holst, H.; Hagenfeldt, L. Increased levels of amino acids in human lumbar and central cerebrospinal fluid after subarachnoid haemorrhage. Acta Neurochir. 1985, 78, 46-56. [CrossRef]

46. Fawcett, J.W.; Asher, R. The glial scar and central nervous system repair. Brain Res. Bull. 1999, 49, 377-391. [CrossRef]

47. Raivich, G.; Bohatschek, M.; Kloss, C.U.; Werner, A.; Jones, L.L.; Kreutzberg, G.W. Neuroglial activation repertoire in the injured brain: Graded response, molecular mechanisms and cues to physiological function. Brain Res. Rev. 1999, 30, 77-105. [CrossRef]

48. Bellapart, J.; Jones, L.; Bandeshe, H.; Boots, R. Plasma Endothelin-1 as Screening Marker for Cerebral Vasospasm After Subarachnoid Hemorrhage. Neurocrit. Care 2013, 20, 77-83. [CrossRef] [PubMed] 